\title{
Transposición Didáctica Del Encadenamiento Basado En Proyectos Ecoeficientes Al Aula-Laboratorio De Química Industrial
}

\author{
Martha Beatriz Ramírez-González \\ Universidad Pedagógica y Tecnológica de Colombia
}

\section{Resumen}

La transposición presentada se realizó sobre el encadenamiento didáctico desarrollado como tesis doctoral de la autora, para la formación en competencias del químico de la Universidad Pedagógica y Tecnológica UPTC, sede Tunja-Colombia. La investigación se abordó con enfoque mixto, de corte longitudinal durante 2017, desde el microcurrículo de la química industrial y basada en las 19 competencias genéricas proclamadas en el Proyecto Alfa Tuning Latinoamérica y 8 competencias específicas construidas endógenamente. El encadenamiento produjo la transformación del imaginario colaborativo de la enseñanza en la Unidad Académica. El producto de la transposición didáctica fue una metodología de autogestión del conocimiento y secuenciación didáctica para desarrollar un proyecto ecoeficiente. Como apropiación social del conocimiento innovador, los químicos upetecistas en formación obtuvieron productos ecoeficientes en el aula-laboratorio de química industrial, caracterizados en cuatro áreas estratégicas, los cuales fueron expuestos y galardonados por su valor agregado, en eventos regionales de innovación y emprendimiento sostenible.

Palabras clave: encadenamiento didáctico, transposición didáctica; noosfera, química industrial ecoeficiente, aprendizaje basado en proyectos ecoeficientes, educación superior.

\section{Introducción}

El encadenamiento didáctico es un constructo elaborado y acuñado por la autora, que encuadra como relación conceptual (similitud y diferencia) del ciclo productivo virtuoso inmerso en un proceso productivo de química industrial, con el proceso educativo de formación en competencias del químico, a partir de la didáctica de las ciencias experimentales, el aprendizaje por investigación (con proyectos ecoeficientes) para la producción de conocimiento innovador, la química verde, el emprendimiento y la sostenibilidad. Desde la investigación educativa, se planteó un conjunto de construcciones basado en la epistemología y redes conceptuales de las ciencias experimentales, y desde lo funcional fue abordada con relaciones didácticas y académicas, mediante la dinámica sociocultural (aula-laboratorio) y sociocientífica del entorno (en la competencia), para movilizar el conocimiento de la química industrial adquirido mediante un aprendizaje desarrollador. El encadenamiento privilegia la articulación de estructuras sucesivas -eslabones- para lograr la adquisición de competencias, a partir de ocho competencias desarrolladas endógenamente para el aprendizaje de la química industrial, distribuidas en 40 características competenciales, bajo la concepción que las competencias no son el fin del proceso de formación, sino el medio para potenciar capacidades complejas a través de estrategias centradas en el estudiante, con transposición desde lo implícito hasta lo explícito, para alcanzar un tipo de conocimiento innovador, con emprendimiento sostenible y una visión transversal del contexto.

La transposición didáctica se basa en los cuatro procesos: selección, reducción, simplificación y reformulación (Ramírez R., 2005) y en éste artículo se instrumentaliza partiendo del objeto de saber científico (química), proyectado hacia el objeto a enseñar (química industrial) y transformándolo en el objeto aprendido, el Proyecto Ecoeficiente. La transposición llevada al espacio físico y conceptual del aula-laboratorio, apropia la metodología de autogestión del conocimiento y aporta los objetos de aprendizaje en química industrial, en forma articulada y sinérgica con los Objetivos del Desarrollo Sostenible.

\section{Referentes Teóricos}

\section{1 ¿Por qué se parte de un encadenamiento?}

En los procesos educativos de producción y transformación de conocimientos en ciencia y tecnología, el constructo sociotécnico de dinámica grupal se reconoce como una cadena de eslabones, porque los resultados obtenidos se logran en cadena; siendo cada eslabón el nodo que soporta y afianza (encadena) las relaciones grupales para lograr el objetivo. 
En el ámbito industrial, una cadena productiva es un círculo productivo, una sucesión de etapas desarrolladas por unidades interconectadas denominadas eslabones, que pueden integrar componentes de diferente origen: físico, tecnológico, humano y geográfico.

El encadenamiento encuadra como un constructo pedagógico, dada la relación conceptual y de semejanza, que representa una espiral productiva en la dinámica sociocultural del aula y sociocientífica del entorno para la formación del químico, competente para asumir los retos emergentes del decenio de 2020, alrededor de la ciencia, tecnología e innovación transformativa.

El circuito productivo o círculo virtuoso que se pretende desarrollar es la formación de profesionales químicos, aplicando estrategias de aprendizaje por investigación y mediado por Aprendizaje Basado en Proyectos Ecoeficientes. El eslabonamiento hacia atrás implica a las relaciones competenciales con el Proyecto Tuning Latinoamérica PTAL (Beneitone, González, \& Wagenaar, 2014) y los principios de la química verde (Anastas \& Warner, 1998); mientras que para Cely \& Ramírez (2017), el eslabonamiento hacia adelante lo constituyen la educación para la sostenibilidad, la pedagogía del emprendimiento y la apropiación social del conocimiento, evidenciado en desarrollos ecoeficientes de innovación transformadora (ver Figura 1).

\section{Figura 1. Constructo de encadenamiento didáctico}

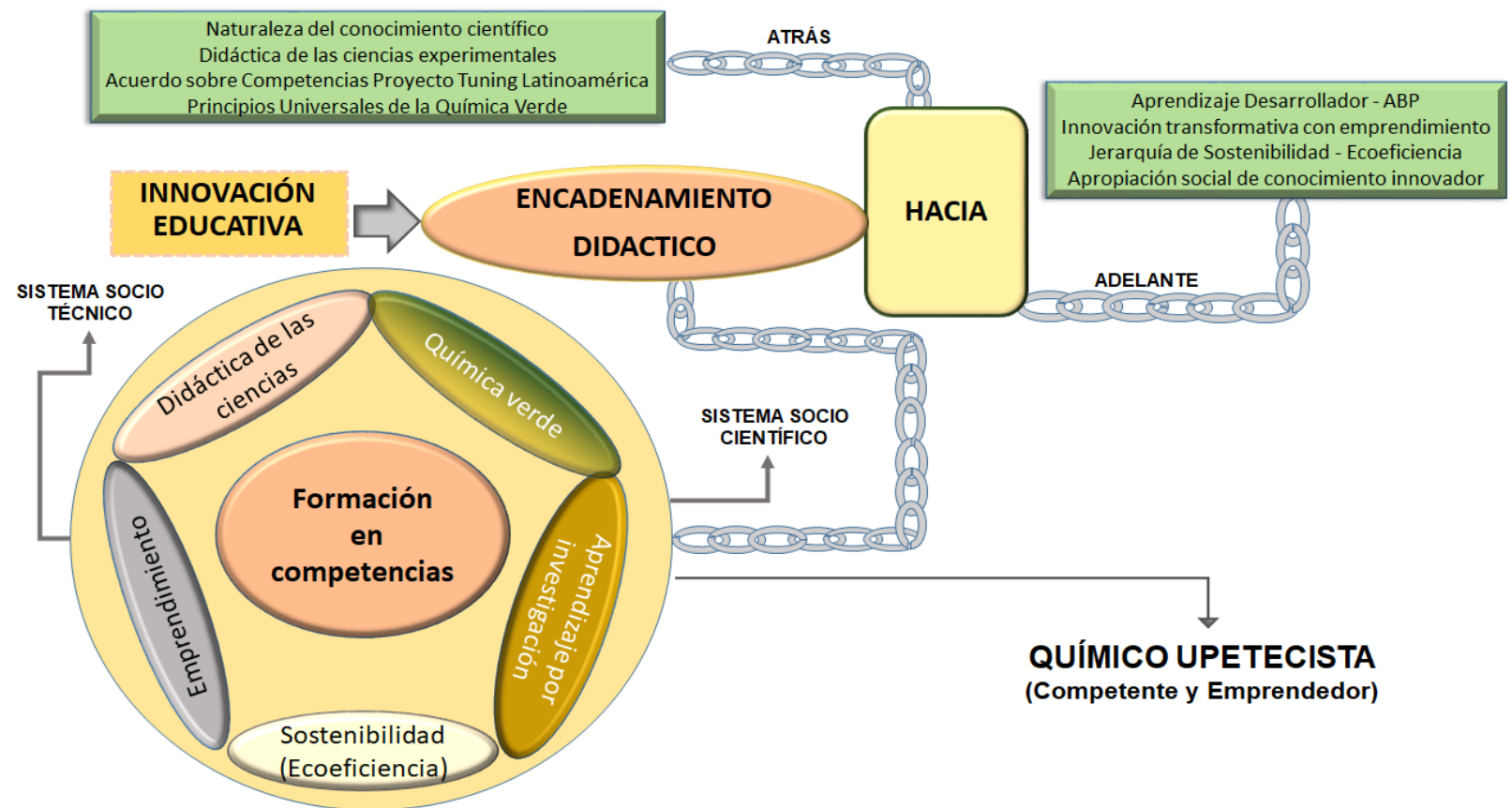

Fuente: elaboración propia

El planteamiento de encadenamiento está fuertemente relacionado con los postulados de la Organización para la Cooperación Económica y el Desarrollo OECD (por su sigla en inglés), que reconoce a las pedagogías y didácticas innovadoras, como orientadoras del conocimiento del mundo real traído por los mismos estudiantes, y que los planes de estudio deben incorporar el conocimiento que los estudiantes comparten, para lo cual se requiere de un nuevo mapeo del entorno de aprendizaje, mediante el denominado "modelo de tecnología educativa de código abierto"(OECD, 2018).

El encadenamiento encuadra en el campo disciplinar didáctico de organización de la acción pedagógica en relación con propuestas innovadoras concretas (Tiberghien, 2008), además dentro del proceso de educación científica con los elementos cognitivos contenidos en la reflexión y autorregulación de las ciencias químicas.

Desde el campo disciplinar de innovación en didáctica de las ciencias, se muestra un camino para crear en el estudiantado una imagen de ciencia dinámica, profundamente holística, distante de las verdades científicas y cercanas a la realidad y complejidad de la actividad científica; en donde, adquiere relevancia el funcionamiento didáctico de Chevallard (1997), plasmado en la teoría de la transposición didáctica, porque aborda la relación didáctica del conocimiento científico y su "paso" o transformación del contenido de saber preciso a una versión didáctica de este objeto del saber, para que hacer posible su aprendizaje. 
En concepto de Gómez-Mendoza (2005), pretende resolver la pregunta central de Chevallard, en el sentido de identificar ¿por qué existen dos flujos de saberes que pasan desde el ambiente hacia el sistema de enseñanza por mediación de la noosfera?

En el encadenamiento, la transposición didáctica constituye un eslabonamiento hacia adelante, porque direcciona el flujo de saberes desde lo implícito a lo explícito, desde lo pre-construido a lo construido, bajo criterios de profundidad y complejidad, del saber sabio al saber pedagógico, con lo cual sea posible hacer las suturas necesarias para juntar las inconexiones teóricas y metodológicas que existen entre investigación científica e investigación educativa (en el aula-laboratorio), cuyo propósito es, Transformación-Aprehensión-Transferencia.

\subsection{La necesidad de la química verde}

Los Principios de Química Verde fueron propuestos originalmente por Paul Anastas \& John Warner (1998), para ser implementados en procesos químicos innovadores, contribuirán a la sostenibilidad del planeta, la sociedad, la economía y el ambiente. La química verde fue adoptada como una propuesta novedosa (en su momento), para reducir o eliminar los problemas ambientales derivados de actividades industriales. Para la Environmental Protection Agency (EPA), la química verde es el "uso de la química para la prevención de la contaminación, y el diseño de productos químicos y procesos benéficos para el ambiente" (Anastas, Kirchhoff, \& Williamson, 2001).

\subsection{La innovación y el emprendimiento}

La innovación es un constructo desarrollado por Joseph Schumpeter en los años 1930, como aquello que se introduce en el mercado con potencial de industrializarlo. El concepto de innovación es amplio y multicontextual, no son solo ideas a granel, sino un todo. En la actividad empresarial, se apunta al avance de la tecnología o hacia las inversiones en las iniciativas de investigación, desarrollo e innovación (I+D+I). La innovación está asociada a un cambio, a una actualización del estado actual en una disciplina o en un ámbito, pero en el campo empresarial implica una nueva función de producción, con potencial para propiciar desarrollo socioeconómico regional; basados en la búsqueda y hallazgo de conocimiento nuevo por investigación, pensamiento crítico y creativo.

La innovación se aplica al proceso como un todo, el producto obtenido y su valor agregado, siempre inherente al espíritu emprendedor propio de la competencia del emprendimiento. El concepto de emprendimiento está referido a la capacidad de una persona para promover esfuerzos adicionales hacia el logro de un objetivo o una meta; posteriormente se asoció a la persona que iniciaba una nueva empresa (emprendedores embrionarios), y en la actualidad es aplicado a personas que sean innovadores o agreguen valor a un producto o proceso ya existente o caracterizado o desarrollado, como resultado de un proyecto o de un plan de negocio. El emprendimiento implica actitud y aptitud de la persona, para impulsar nuevos retos, nuevos proyectos, que le permita avanzar paulatinamente hacia la consecución del resultado propuesto, para la satisfacción de necesidades profesionales o empresariales, lo cual requiere de un elemento vital denominado espíritu emprendedor (Varela, 2010).

La iniciativa es esencial en la actividad de los emprendedores, entendida como proceso y capacidades, y supone la búsqueda de resultados inciertos originados en la experiencia. A diferencia de la creatividad, que puede ser desordenada, la innovación no lo es. Para Nueno (2009), el emprendedor descubre oportunidades donde los demás no ven nada, aunque no todo creativo es exitoso en la ejecución de ideas empresariales. En la actualidad, la demanda en empresas se concentra en competencias de emprendimiento e innovación, en personas capaces de emprender, y no solo en el sentido de crear una empresa, sino en la capacidad de innovar en todas aquellas tareas que su desempeño profesional requiera; sin embargo, a pesar de la necesidad de potenciar el emprendimiento, son pocos los casos exitosos de transposición de ésta competencia (Segarra, Grangel, Belmonte, \& Aguado, 2017). En este sentido, las competencias emprendedoras son todas aquellas capacidades, destrezas, aptitudes, habilidades que posibiliten al emprendedor desempeñarse idóneamente en la realización de actividades sistémicas de emprendimiento y en la resolución por integración del saber hacer, saber conocer y saber ser en su contexto sociocultural y sociocientífico de la química industrial.

\subsection{La ecoeficiencia actitud responsable con el desarrollo sostenible}

La ecoeficiencia es un concepto universal que implica la relación entre el impacto ambiental añadido y el valor agregado al proceso, apuntando a lograr mejores productos y servicios con un menor uso de recursos y la reducción del flujo de residuos y emisiones; que en términos termodinámicos optimiza el uso eficiente de recursos y reduce la destrucción de exergía. 
La ecoeficiencia es una forma práctica y aún innovadora filosofía de producción limpia, en la cual se considera al sistema industrial como ecosistema que posibilita la autogestión anergética. La ecoeficiencia, pretende responder a las necesidades de particulares y de empresas, que buscan alcanzar la sostenibilidad bajo el principio rector con desafíos más globales e integrados, como: agotamiento de recursos naturales, contaminación y cambio climático; caracterizados y cuantificados mediante balances de energía y exergía. En química industrial, la eficiencia energética es un aporte conceptual relevante para la sostenibilidad, pero su implementación es viable cuando se acompaña con un encadenamiento hacia adelante hacia un proceso integrado de transformación limpia con patrones de consumo racional de energía y recursos naturales, ajustando el coeficiente químico-verde "economía del proceso", sin que se genere el efecto rebote (Efecto Jevons), ni directo ni indirecto. La formación de un químico desde el compromiso científico del desarrollo sostenible, educación para la sostenibilidad y la estructura de un pensamiento complejo alrededor de los sistemas de producción sostenible, el agotamiento de los recursos naturales y su uso racional con responsabilidad social, para superar la resistencia al cambio.

\subsection{El aprendizaje desarrollador en la didáctica}

Para González (2018), el aprendizaje desarrollador es el proceso de apropiación que tiene el estudiante de la cultura, bajo condiciones de orientación e interacción social. Para lograr un aprendizaje desarrollador es necesario que el aprendizaje empodere al estudiante como un ser activo y regulado, co-constructor de su propio conocimiento y protagonista, estimulando su creatividad y la transferencia de conocimientos y habilidades a escenarios reales, lo que se traduce en aprendizaje como producción de sus propios y nuevos. Es decir, conocimientos sobre su propio proceso cognoscitivo, para potenciar la necesidad de aprender a aprender.

El aprendizaje desarrollador garantiza al individuo una apropiación activa y creadora de la cultura, propiciando el desarrollo de su auto perfeccionamiento constante, de su autonomía y autodeterminación, en íntima conexión con los procesos de socialización, compromiso y responsabilidad social (Lima, 2005).

La relevancia del aprendizaje basado en proyectos ABP como mediador en el proceso de aprendizaje del estudiante, favorece su acercamiento al contexto profesional y el mercado de destino; sin embargo, se puede atravesar inapropiadamente la delgada línea y sesgarse a procesos de formación para el trabajo como técnico o tecnólogo en lugar de un profesional en química. Esto es evitable, cuando se planifican correctamente los niveles de competencia (saber-hacer), los distintos grados de inmersión profesional (propuestas y proyectos) a desarrollar; teniendo precaución de no caer en la resolución de problemas irrelevantes o artificiales, cuya única referencia sea intra-académica (Ramírez \& Cely, 2017).

Los escenarios de la mediación deben planificarse con minucia, para intervenir situaciones reales bien documentadas o con acceso para ello, evitando abordar problemas insuficientemente contextualizados, que evadan la complejidad e incertidumbre propias de condiciones y requerimientos de situaciones profesionales reales. La prioridad es, minimizar el sesgo de caer en emulaciones-simulaciones triviales que solo son teóricas.

\section{Metodología}

- La investigación se abordó con un enfoque mixto, aplicando los criterios propuestos por Tashakkori \& Teddlie (2003) y Creswell (2009). La combinación de los métodos cualitativo, facilita la aproximación a un problema desde diferentes métodos y como investigación educativa, implica una triangulación metodológica (Aguilar \& Barroso, 2015).

- Dada las condiciones de la noosfera, el contexto sociocientífico y sociocultural universitario, la investigación educativa debe zanjar la brecha de crear teorías sobre educación, inconexas con la práctica educativa, principalmente a nivel de aula-laboratorio (Lederman, 2018).

- La temporalidad fue de dos semestres académicos consecutivos, durante el año 2017.

- La población estuvo constituida por treinta y ocho (38) estudiantes que cursaron la asignatura química industrial (octavo semestre) de pregrado en Química de la UPTC y discriminados así: 24 de género femenino $(63,15 \%)$ y 14 de género masculino $(36.85 \%)$.

- Cabe señalar que en éste artículo se realiza la transposición didáctica del encadenamiento realizado en el lapso 2014-2016, mediante una fase anterior (publicada en Ramírez M. B., 2018), en donde se presentan la formación en competencias del químico, con mediación pedagógica del ABP y sobre la construcción endógena de las siguientes ocho (8) competencias específicas:CE1: Habilidades interpersonales; CE2: Trabajo en equipo; CE3: Análisis de relaciones conceptuales; CE4: Manejo fuentes y análisis de información; CE5: Pensamiento lógico-conceptual; CE6: Innovación; CE7: Emprendimiento; CE8: Comunicación científica. 
- La movilización del conocimiento científico, a través del aprendizaje por investigación, la química verde, el emprendimiento, la ecoeficiencia, la innovación transformativa y los Objetivos del Desarrollo Sostenible ODS, proclamados en la Agenda 2030 (Colciencias, 2018).

- Los supuestos básicos usados para determinar las operaciones de la transposición, son: los objetivos de aprendizaje; los saberes previos y necesidades de los aprendizajes; el reconocimiento de las necesidades y noosfera (contexto de aprendizaje); intenciones socioculturales, políticas e ideológicas; aprendizaje y significatividad lógica (Ramírez R., 2005).

- Los procesos metodológicos desarrollados fueron: clasificar y depurar para la selección; condensar e integrar para la reducción; accesibilidad y comprensibilidad para la simplificación; perfectibilidad para la reformulación de los recursos didácticos.

\section{Resultados}

En la sociedad actual de información y conocimiento, su generación, procesamiento, transmisión y transferencia, son fuente fundamental de productividad, por su capacidad de crear, desarrollar, difundir y explotar el conocimiento, para incrementar la capacidad competitiva.

Asi mismo, la gestión del conocimiento es un proceso lógico, organizado y sistemático para producir, transferir y aplicar en situaciones concretas una combinación armónica de saberes, experiencias, información contextual y apreciaciones expertas que reproducen nueva información.

Mediante los procesos de selección, reducción, simplificación y reformulación, propios de la transposición didáctica (Ramírez R., 2005), fue posible construir secuencialmente elementos didácticos, para instrumentalizar el aprendizaje la química industrial ecoeficiente.

\subsection{Metodología para la autogestión del conocimiento}

Este resultado es un producto tecnológico blando, que contribuyó a redireccionar la dinámica social en la formación profesional de un químico, con el fin de alcanzar los objetivos determinados y determinantes en los lineamientos curriculares, el cual agrega valor al estado actual del conocimiento en didáctica de las ciencias químicas. Consiste en una metodología sistemática de planeación y autogestión en el microcurrículo de química industrial (para 16 semanas lectivas del semestre académico), consta de un conjunto de fases, etapas, barreras de entrada, acciones estratégicas para superar dichas barreras, momentos de, y en la práctica educativa del aulalaboratorio, los instrumentos para la evaluación auténtica de competencias, con base en la triangulación metodológica (cualitativa-cuantitativa), la recensión de un documento final consolidado y el portafolio de evidencia (ver Tabla 1).

Para efectos del proceso de reducción y simplificación, en concordancia con Hjerensen y otros (2000), Cann (2009), Obaya \& Vargas (2013), se integraron recursos conceptuales para apropiar los criterios que faciliten cuantificar, qué tan amigable es una actividad experimental de química con el medio; por lo tanto, se reformuló en términos de análisis comparativo entre las buenas prácticas con química verde vs. la experiencia tradicional. 
Tabla 1. Metodología de autogestión construida para la transposición didáctica del encadenamiento

\begin{tabular}{|c|c|c|c|c|c|c|c|c|c|c|}
\hline \multicolumn{2}{|c|}{ FASES } & \multicolumn{2}{|c|}{ ACTOR } & \multirow{2}{*}{ ETAPA } & \multirow{2}{*}{ 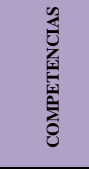 } & \multirow{2}{*}{ BARRERAS DE ENTRADA } & \multirow{2}{*}{ ACCIONES } & \multirow{2}{*}{$\begin{array}{l}\text { CUÁNDO } \\
\begin{array}{l}\text { Semana Acadé } \\
\text { mica }\end{array}\end{array}$} & \multicolumn{2}{|c|}{ CRITERIOS DE EVALUACIÓN } \\
\hline & $\mathbf{E}$ & D & $\mathbf{A}$ & & & & & & $\begin{array}{l}\text { CUALITA } \\
\text { TIVA }\end{array}$ & CUANTITATIVA \\
\hline 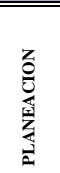 & $\mathrm{x}$ & $\mathrm{x}$ & & $\begin{array}{l}\text {-Identificicación de problemas. } \\
\text {-Soporte Interdisciplinar en } \\
\text { Emprendimiento. }\end{array}$ & $\begin{array}{l}\text { 1G, 6G, } \\
\text { CE3, CE6, } \\
\text { CE7 }\end{array}$ & $\begin{array}{l}\text {-Desconoci-miento de la } \\
\text { pedagogía. } \\
\text {-Temor al cambio. } \\
\text {-Poca autonomía. } \\
\text {-No conocen el sector real. } \\
\text { - Baja confianza entre } \\
\text { compañeros. }\end{array}$ & $\begin{array}{l}\text {-Cualificación en formulación y } \\
\text { en emprendimiento sostenible. } \\
\text {-Fortalecimiento de química } \\
\text { verde. } \\
\text {-Motivación para trabajo en } \\
\text { equipo }\end{array}$ & 1 y 2 & $\begin{array}{l}\text {-Discusión plenaria. } \\
\text {-Conciliación de los } \\
\text { temas y alcances de } \\
\text { investigación. }\end{array}$ & $\begin{array}{l}\text { Tres propuestas de } \\
\text { perfil de proyecto, en } \\
\text { informe escrito. }\end{array}$ \\
\hline \multirow{5}{*}{ 孞 } & $\mathrm{x}$ & $\mathrm{x}$ & $\mathrm{x}$ & $\begin{array}{l}1^{\mathrm{a}} \text { Etapa. } \\
\text { Selección del problema }\end{array}$ & $\begin{array}{l}4 \mathrm{G}, 14 \mathrm{G}, \\
17 \mathrm{G}, \mathrm{CE} 3 \\
\mathrm{CE} 7\end{array}$ & $\begin{array}{l}\text {-Baja autoestima. } \\
\text {-Inseguridad. } \\
\text {-Visión fáctica, no de largo } \\
\text { plazo. } \\
\text {-Inexperiencia e inseguridad en } \\
\text { este trabajo. } \\
\text {-Debilidad revisión } \\
\text { bibliográfica. } \\
\end{array}$ & $\begin{array}{l}\text {-Grupo de trabajo colaborativo. } \\
\text {-Matrices de decisión. } \\
\text {-Soluciones sostenibles. } \\
\text {-Empoderamiento } \\
\text {-Actualización en bases de datos. }\end{array}$ & 3 y 4 & $\begin{array}{l}\text {-Mapa conceptual. } \\
\text {-Diagrama de flujo. } \\
\text {-Diagrama del proceso } \\
\text { a realizar en el } \\
\text { laboratorio. }\end{array}$ & $\begin{array}{l}\text { Instrumento de } \\
\text { coevaluación } \\
\text { Exposición de } \\
\text { socialización en } \\
\text { plenaria del curso. }\end{array}$ \\
\hline & $\mathrm{x}$ & $\mathrm{x}$ & $\mathrm{x}$ & $\begin{array}{l}2^{\mathrm{a}} \mathbf{E t a p a} \\
\text { Diseño del proceso investigativo }\end{array}$ & $\begin{array}{l}\text { 1G, 2G, } \\
18 \mathrm{G} 13 \mathrm{G} \\
17 \mathrm{G}, \mathrm{CE} 4 \\
\text { CE6, CE7 }\end{array}$ & $\begin{array}{l}\text {-No apropia el rol como } \\
\text { investigador ni su ritmo de } \\
\text { trabajo. } \\
\text {-No auto-reflexiona } \\
\text { objetivamente sobre su trabajo. }\end{array}$ & $\begin{array}{l}\text {-Planeación de materiales, } \\
\text { métodos, tiempos reales y } \\
\text { ociosos, \# ensayos, movimientos, } \\
\text { ruta de residuos y carga } \\
\text { contaminante. } \\
\text { Retroalimentación. }\end{array}$ & 5 a 8 & $\begin{array}{l}\text {-Autoeva- } \\
\text { luación } \\
\text {-Heteroeva- } \\
\text { luación }\end{array}$ & $\begin{array}{l}\text { Informe consolidado de } \\
\text { actividades de la etapa. } \\
\text { Formato diligenciado } \\
\text { con reactivos, material } \\
\text { y equipos. }\end{array}$ \\
\hline & $\mathrm{x}$ & $\mathrm{x}$ & $\mathrm{x}$ & $\begin{array}{l}3^{\mathrm{a}} \text { Etapa. } \\
\text { Desarrollo experimental }\end{array}$ & $\begin{array}{l}\text { 17G, 19G } \\
\text { CE1, CE2, } \\
\text { CE4, CE6 }\end{array}$ & $\begin{array}{l}\text {-Planeación vs plan de trabajo. } \\
\text {-No se autorregula } \\
\text { objetivamente sobre su trabajo } \\
\text { desarrollado. }\end{array}$ & $\begin{array}{l}\text {-Reprogramación. } \\
\text {-Aplicar mejoras, validar datos, } \\
\text { retro- alimentar. } \\
\text {-Nuevo diagnóstico. }\end{array}$ & 5 a 8 & $\begin{array}{l}\text {-Resumen Analítico } \\
\text { Especiali } \\
\text { zado } \\
\text {-Innovación en } \\
\text { metodologías. }\end{array}$ & $\begin{array}{l}\text { Informe consolidado de } \\
\text { avance, según } \\
\text { metodología. }\end{array}$ \\
\hline & $\mathrm{x}$ & $\mathrm{x}$ & & $\begin{array}{l}4^{\text {a }} \text { Etapa. } \\
\text { Desarrollo ecoeficiente }\end{array}$ & $\begin{array}{l}\text { 8G, 9G, } \\
\text { 17G, CE5, } \\
\text { CE6, CE7 }\end{array}$ & $\begin{array}{l}\text {-Los estudiantes muestran poco } \\
\text { interés por la producción más } \\
\text { limpia y los estándares de las } \\
\text { Normas ISO. }\end{array}$ & $\begin{array}{l}\text {-Aspectos positivos, negativos, } \\
\text { mejoras. }\end{array}$ & 5 a 16 & $\begin{array}{l}- \text {-Revisión por parte } \\
\text { del docente } \\
\text { responsable del curso. }\end{array}$ & $\begin{array}{l}\text { Ruta para la mitigación } \\
\text { de los residuos } \\
\text { generados. } \\
\text { Coevaluación del } \\
\text { proceso. }\end{array}$ \\
\hline & $\mathrm{x}$ & $\mathrm{x}$ & & $\begin{array}{l}5^{\text {a }} \text { Etapa. } \\
\text { Plan de negocio. } \\
\text { Factibilidad integrada }\end{array}$ & $\begin{array}{l}5 \mathrm{G}, 17 \mathrm{G} \\
19 \mathrm{G}, \mathrm{CE} 4 \\
\text { CE7 }\end{array}$ & $\begin{array}{l}\text {-Desconoci-miento del } \\
\text { mercadeo y gestión } \\
\text {-Desinterés por el área } \\
\text { económica y financiera. }\end{array}$ & $\begin{array}{l}\text {-Orientación sobre el área } \\
\text { económica, financiera, costeo y de } \\
\text { responsabilidad social. }\end{array}$ & 14 a 16 & & $\begin{array}{l}\text { Documento escrito con } \\
\text { resultados y producto } \\
\text { con estándares y } \\
\text { requerímientos del } \\
\text { INVIMA }\end{array}$ \\
\hline \multirow[t]{2}{*}{ 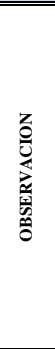 } & $\mathrm{x}$ & $\mathrm{x}$ & & Validación interna & $\begin{array}{l}5 \mathrm{G}, 12 \mathrm{G} \\
\text { CE5, CE6 }\end{array}$ & $\begin{array}{l}\text { - Poco conocimiento (teorías) y } \\
\text { sin mejora inmediata de la } \\
\text { realidad concreta (práctica). } \\
\text { Desinterés por nuevas } \\
\text { actualizaciones de Normas ISO } \\
\text { y tecnologías emergentes }\end{array}$ & $\begin{array}{l}\text {-Recopilación de la información. } \\
\text {-Interpretación, validación, e } \\
\text { integración de datos } \\
\text {-Modifica } \\
\text {-redefine la recensión, póster y } \\
\text { presentación. } \\
\text {-Video construido durante el } \\
\text { proceso de construcción y gestión } \\
\text { del conocimiento. }\end{array}$ & 16 & $\begin{array}{l}\text {-Compe } \\
\text { tencia comunicativa, } \\
\text { oral y visual de } \\
\text { resultados, } \\
\text { conclusiones y aportes } \\
\text { a futuros trabajos. } \\
\text { Presentación póster y } \\
\text { exposición con Power } \\
\text { Point o Prezi. }\end{array}$ & $\begin{array}{l}\text { Plan de negocio, } \\
\text { constituido por } \\
\text { indicadores } \\
\text { económicos, } \\
\text { financieros, } \\
\text { ambientales, sociales. } \\
\text { Portafolio de } \\
\text { evidencias. } \\
\text { Heteroevaluación } \\
\text { mediante rúbrica } \\
\text { integrada, consolidada. }\end{array}$ \\
\hline & $*$ & $*$ & $*$ & Validación Externa & $\begin{array}{l}\text { 5G, 12G, } \\
\text { CE6, CE7, } \\
\text { CE8 }\end{array}$ & $\begin{array}{l}\text {-Inseguridad para interactuar } \\
\text { con empresarios y sector } \\
\text { externo, en escenarios físicos } \\
\text { extramurales. }\end{array}$ & $\begin{array}{l}\text { - Replicar el estudio y llegar a los } \\
\text { mismos resultados. }\end{array}$ & 17 & $\begin{array}{l}\text { Eventos externos: } \\
\text { ferias de innovación, } \\
\text { de emprendi-miento. }\end{array}$ & $\begin{array}{l}\text { *Empresario, público } \\
\text { asistente y los } \\
\text { empresarios jurados del } \\
\text { evento y ángeles } \\
\text { inversores de Silicon } \\
\end{array}$ \\
\hline 辇 & $\mathrm{x}$ & $\mathrm{x}$ & & Argumentación de trabajo. & & $\begin{array}{l}\text {-Ferias empresariales } \\
\text {-Autodetermina-ción, } \\
\text { optimismo, liderazgo, tenacidad. } \\
\text { - Innovación. } \\
\text { - Emprendi- } \\
\text { miento }\end{array}$ & $\begin{array}{l}\text { Reflexión crítica. } \\
\text {. }\end{array}$ & 17 & $\begin{array}{l}\text { Indicadores medibles } \\
\text { relevantes vinculantes } \\
\text { a la ecoeficiencia. } \\
\text { Pertinencia-fiabilidad } \\
\text { Competencia } \\
\text { comunicativa. }\end{array}$ & $\begin{array}{l}\text { Autoevalua } \\
\text { ción Heteroevaluación. } \\
\text { Coevalua } \\
\text { ción. }\end{array}$ \\
\hline
\end{tabular}

\subsection{Secuenciación en la transposición.}

La programación del aula-laboratorio es una secuencia de elementos didácticos que se presenta a través de etapas, roles e importancia del eslabonamiento hacia atrás y hacia adelante, para la transposición didáctica del proceso de formación en competencias (ver Figura 2). 


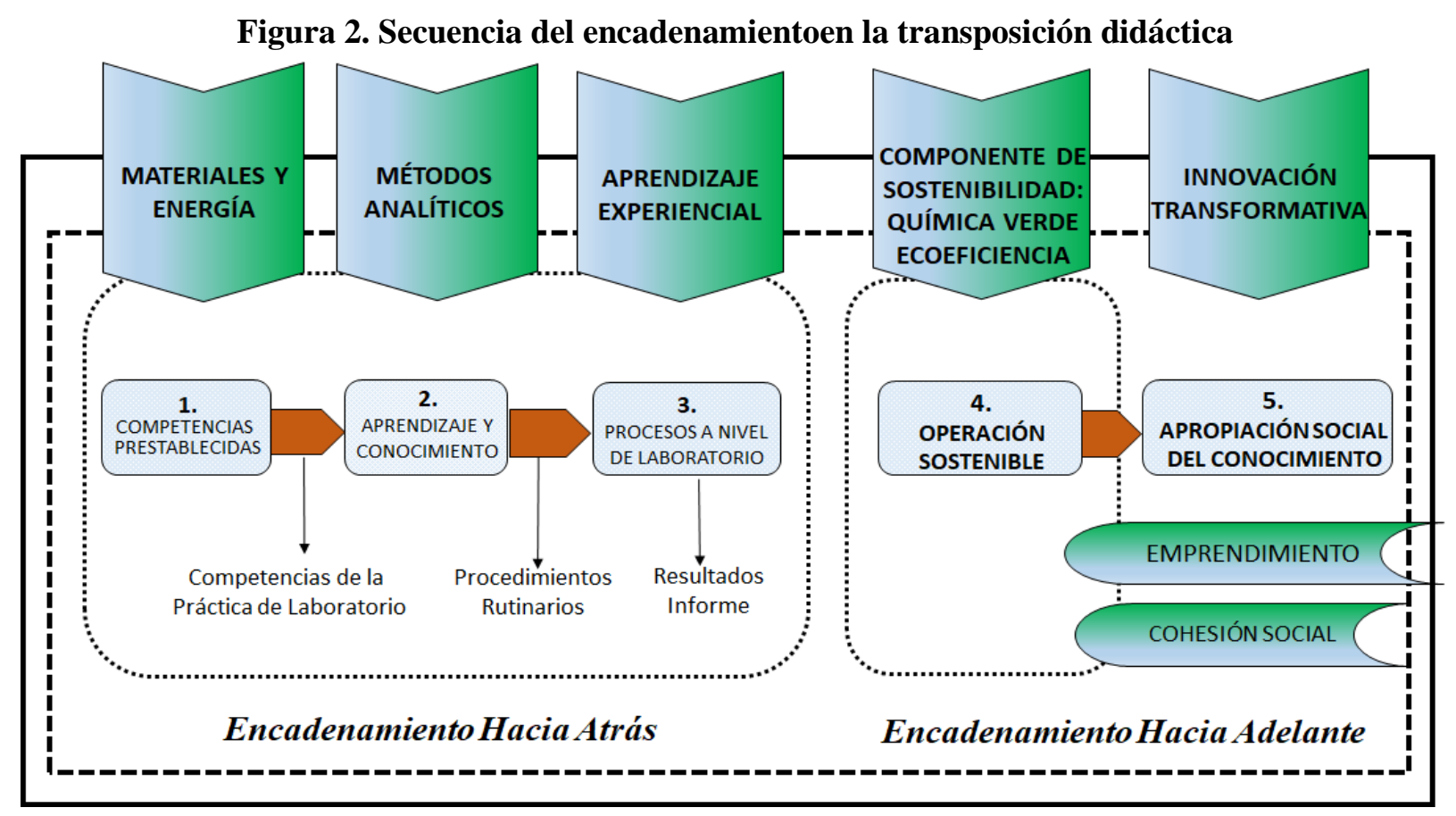

Fuente: Ramírez-González (2018)

Selección didáctica. Corresponde al encadenamiento hacia atrás, dado que, son los principios y teorías correspondientes a los objetivos de la química emergente del decenio 2020 de y a las condiciones socioculturales del futuro químico upetecista.

Reducción didáctica. Corresponde al encadenamiento hacia adelante (eslabón de operación sostenible), en donde los estudiantes no se entrenan para resolver una guía, sino que son cualificados en la noosfera del sistema productivo sostenible, necesario la comprensión y acción (producción) en el aula-laboratorio. De acuerdo con las perspectivas didácticas del contexto, las áreas fueron: tiempos y movimientos, planificación de materiales, administración del inventario, ruta crítica para el desarrollo de proyectos.

Simplificación didáctica. Esta operación permite asumir que la realidad puede describirse mediante signos más simples que los que presenta el saber científico, corresponde a la transformación ecoeficiente en el aulalaboratorio de química industrial, bajo los propósitos de la innovación y los objetivos del desarrollo sostenible, que caracterizan la producción limpia y productos ambientalmente sanos. Al proceso didáctico se incorporan los estándares internacionales de los sistemas de gestión (asociados a la Norma ISO para entornos industriales) para el aseguramiento de la calidad, en éste caso de la producción y productos ecoeficientes obtenidos en el aulalaboratorio.

El concepto de producción industrial conforme con la norma, se apropia y aplica, bajo las condiciones y limitaciones de una producción embrionaria a nivel del aula-laboratorio; es decir, el cumplimiento en el contexto industrial, de pautas universales de estandarización del proceso con calidad técnica, ambiental, de seguridad en el trabajo y de eficiencia energética sostenible. Ésta característica competencial agrega valor al encadenamiento para la formación por competencias, porque arraiga la cultura del estándar del producto, proceso y calidad (laboratorio).

Como resultados de la simplificación didáctica, los estudiantes adquieren y movilizan sus conocimientos hacia la calidad, seguridad, gestión y desarrollo sostenible, contemplados en las normas ISO, mediante valoración de la intangible calidad, factor determinante para proclamar que un producto está terminado. Aquí se incluye la gestión y ruta de residuos, como cultura, filosofía y compromiso de la producción ecoeficiente, para minimizarlos y para mitigar su impacto negativo cuando se produzcan, mediante y biotecnología para su descontaminación o inactivación química.

Reformulación didáctica. Esta operación conduce a la reescritura del libreto y discurso científico de la ciencia química, hacia un discurso pedagógico en términos de contenidos enseñables para reconstruir el saber hacia la química sostenible, reestructurando el paradigma ecoeficiente en la investigación química (ver Figura 3). 
La pirámide del valor presenta de manera diáfana la sostenibilidad, determinada por la jerarquía ecoeficiente requerida para el químico del decenio 2020 y su emprendimiento sostenible, con lo cual se converge en la generación del conocimiento innovador, su apropiación social y el desarrollo de empresas embrionarias basadas en el conocimiento, empresas spin-off.

Figura 3. Reformulación didáctica: pirámide del valor del encadenamiento

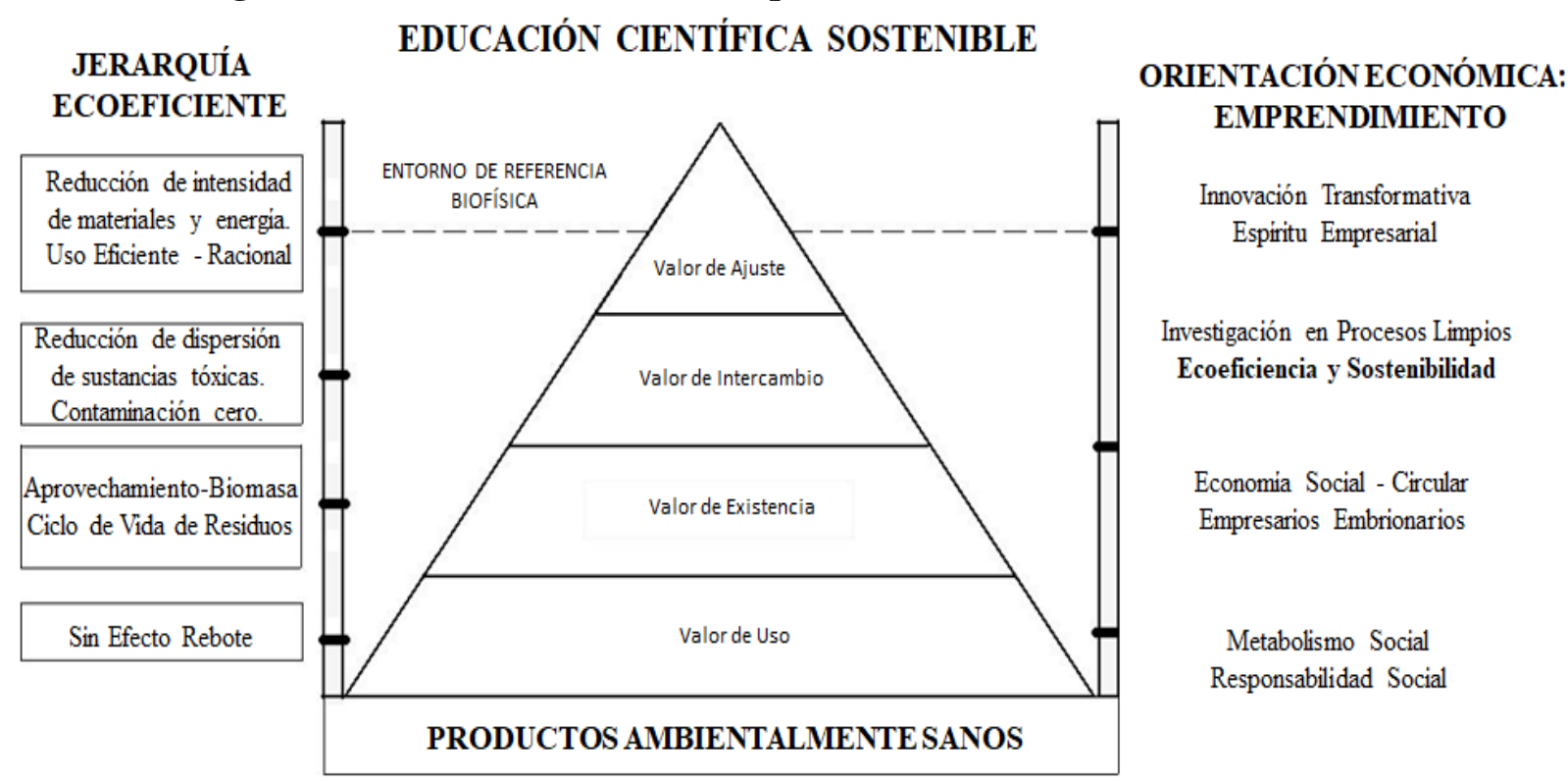

Fuente: Elaboración propia, adaptado de Cely-Niño (2017)

\subsection{Reformulación del proyecto para un enfoque ecoeficiente}

El proyecto ecoeficiente es aquel que cumple con los siete principios proclamados por el Consejo Mundial de Ecoeficiencia, es decir, que posea intrínsecamente la jerarquía de: prevención, reuso, reciclado, recuperación de materiales, mejora de la productividad, ahorro de energía y materias primas, reducción de residuos y materiales tóxicos, disminución de riesgos, ahorro en el gasto de control de la contaminación, mejores medidas de sanidad y seguridad, reducción de los riesgos civiles ambientales.

En la química industrial, la ecoeficiencia, juega un destacado papel, porque los procesos que se desarrollan al interior de la asignatura, están relacionados con la transformación industrial a base de las ciencias químicas, la fisicoquímica, la cinética y la termodinámica del equilibrio. Por lo tanto, un proyecto ecoeficiente incorpora cultura, criterios, estrategias, acciones de producción limpia, respeto por el ambiente con responsabilidad social empresarial, erigiéndolo como aprendizaje desarrollador porque propicia el auto perfeccionamiento constante, la autonomía y la autodeterminación, al cumplir los tres criterios esenciales descritos.

En la Figura 4 se exhibe el diagrama de flujo que se desarrolló, constitutivo de un proyecto a partir del cual no solo se resuelve un problema real, sino que genera un producto ecoeficiente con indicadores de ecoinnovación, lo cual constituye el mediador pedagógico apropiado para la transposición didáctica del encadenamiento para generar competencias en química industrial. 
Figura 4. Producción de proyectos ecoeficientes en el aula-laboratorio de química industrial

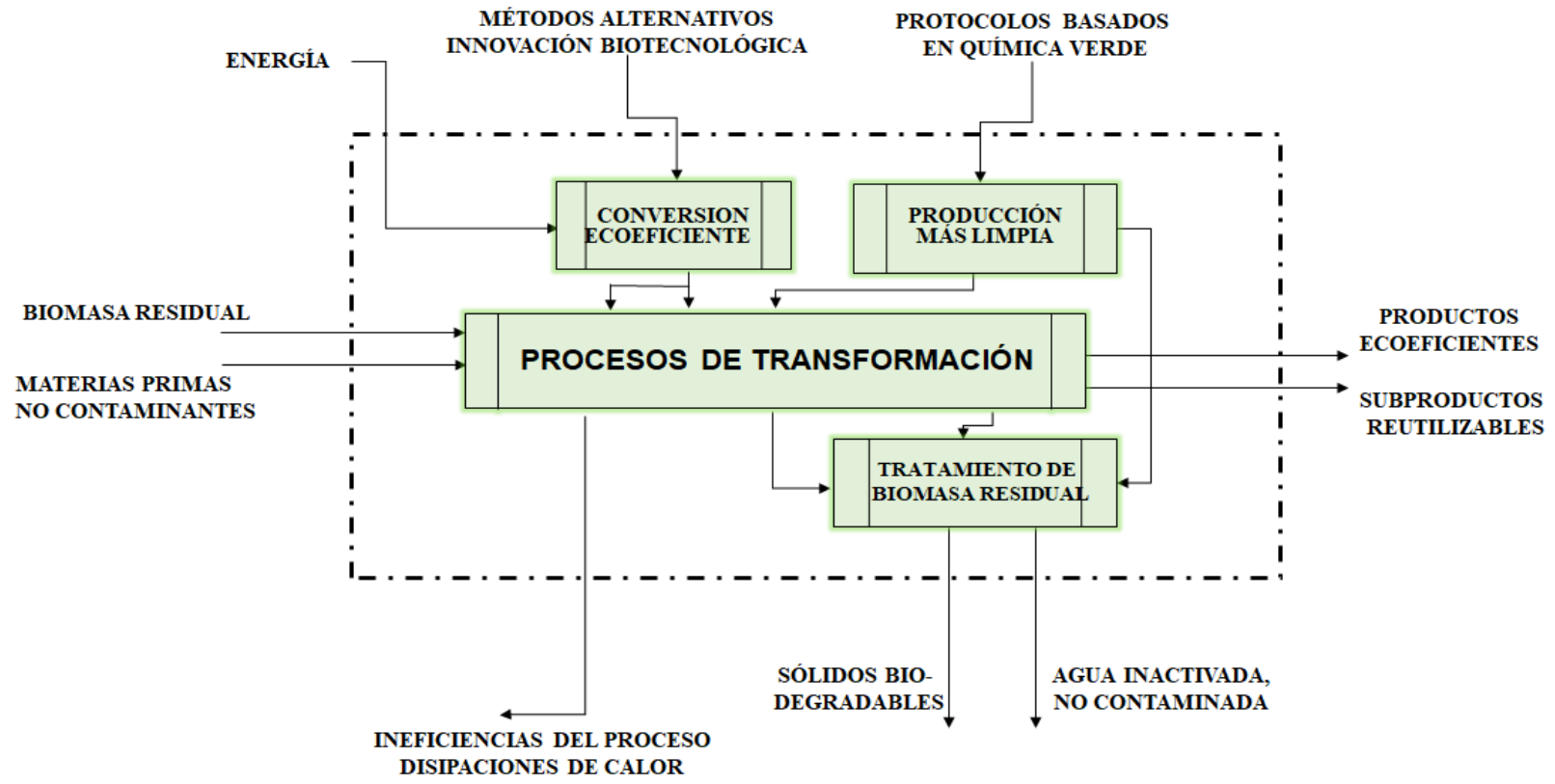

Fuente: Ramírez-González (2018)

El proyecto ecoeficiente que se desarrolle con ésta metodología posee un papel instrumental a través de los ecoindicadores que identifican, calculan y miden los factores de criticidad, en etapas y equipos del proceso con mayores ineficiencias. Las ineficiencias corresponden a pérdidas de exergía (anergía) que pueden generar impactos negativos severos sobre el medio ambiente; tales como dependencia de líquidos volátiles y gases que deterioran la capa de ozono, efluentes y residuos sólidos, como difusión másica ante escapes, roturas o descompensación en líneas de flujo.

\section{4. Áreas y recursos didácticos desarrollados en la transposición}

De acuerdo con lo descrito en las secciones anteriores, la reformulación se realizó mediante un proceso secuencial autónomo, flexible y autorregulado, en el cual se modificaron las políticas, protocolos y tipología de la planeación, acción y comunicación de los resultados obtenidos de la experiencia verde y ecoeficiente y sostenible en el aula-laboratorio. Los cuatro (4) recursos didácticos generados, mediante los cuales se redirecciona el conjunto de criterios y pautas para el trabajo en el aula-laboratorio de química industrial verde, que de acuerdo con Soussan (2003), son promisorios para estructurar o adaptar el conocimiento científico y transferirlo como conocimiento innovador para retroalimentar el proceso de aprendizaje.

En su orden, los cuatro objetos de conocimiento reformulados fueron: Principios de Química Verde aplicados al aprendizaje experimental de la química industrial ecoeficiente; planeación y requisición semestral de materiales para aprendizaje experiencial; pautas para construcción del diagrama de proceso en química industrial verde; criterios para reportes en sesión de laboratorio en química industrial verde.

Como en cualquier actividad de la ciencia experimental, el componente de ensayo y error ocupó un papel primordial y, en virtud de un análisis in situ y de resultados del encadenamiento previo, se acotaron las áreas, temas y trabajos, para cumplir con la selección, reducción y simplificación, esto es, desarrollar y emprender aquellos proyectos que respondan a los cinco Objetivos del Desarrollo Sostenible y cumplan con los siete requisitos de ecoeficiencia, prioritarias para la formación del químico para el decenio de 2020 (Cely \& Ramírez, 2017).

Las cuatro áreas de trabajo como estratégicas para los procesos inmersos en la transposición: fitoquímica (productos naturales), agroindustria, alimentos y biotecnología; las cuales cumplen con los propósitos de la transposición del encadenamiento didáctico: (a) los proyectos son resultado de un proceso de aprendizaje por investigación, (b) están orientadas a transformación de materiales vegetales de la región y de su biomasa residual, (c) su emprendimiento está dirigido hacia la bioempresa, por tratarse de soluciones biotecnológicas. 


\section{Conclusiones}

En la transposición didáctica desarrollada, del proceso de formación en competencias del químico, se modificó el modus operandi de la enseñanza instruccional hacia el desarrollo de las clases con la realización de proyectos, que permitieron integrar teoría, práctica y trabajo colaborativo, reflejando la aplicabilidad de conocimientos de las diferentes áreas de la química industrial, adquiridos a través de la formación académica recibida. El Proyecto Ecoeficiente, usado como aprendizaje desarrollador, integra los propósitos de la formación en competencias, en sinergia con métodos, procedimientos y productos a obtener (evidencias) en el aula-laboratorio, en el horizonte de tiempo semestral (16 semanas), lo cual posibilita replantear y mejorar en forma continua la didáctica de la ciencia química experimental.

Desde la acción didáctica, el encadenamiento permitió modificar el paradigma estudiantil que presuponía una práctica en el laboratorio como el conjunto de pasos para la recopilación de datos cuantitativos, inconexos con el aprendizaje, a pesar que con ellos es posible la resolución de preguntas que nunca formuló el estudiante. La transformación lograda, permite que la experiencia en el laboratorio sea una práctica significativa, imbricada como una actividad mental que exige análisis y razonamiento para generar alternativas, diferente al listado de respuestas esperadas a una guía repetitiva semestre a semestre; en otras palabras, un escenario en donde la acción práctica se transforma de manera auténtica en aprendizaje por investigación, reflexión profunda, en lugar de evaluación de una tarea cumplida, lo cual fue decisivo para potenciar competencias de autodesarrollo y autogestión del conocimiento.

Los estudiantes aprendieron a aprender los conocimientos científicos de la química industrial y los apropiaron de forma dinámica, incorporando los postulados universales de la innovación transformativa y el emprendimiento sostenible como fuente de empoderamiento, que les permitió la producción de conocimiento innovado y aplicado, el cual pudieron movilizar a través de la apropiación y divulgación del conocimiento. Los productos obtenidos de la transposición didáctica (en promedio 10 por semestre), se organizaron y acreditaron en portafolios de evidencias, a través de: proyectos emprendidos y productos ecoeficientes, expuestos en ferias regionales y cinco ponencias internacionales en tres eventos internacionales. Los cuales fueron seleccionados y galardonados por el sector productivo, los inversores y las organizaciones cofinanciadores de desarrollos innovadores de alto valor agregado en las áreas eco-eficientemente estratégicas: agroindustria, productos naturales, alimentos y biotecnología, promisorias para futuras empresas de base tecnológica.

\section{Agradecimientos}

La autora expresa sus agradecimientos al Grupo de Investigación en Ecoeficiencia, Productos Naturales e Innovación Tecnológica de la Universidad Pedagógica y Tecnológica de Colombia. A su Director, Profesor Víctor Hugo Cely Niño, PhD.

\section{Referencias}

Aguilar, S., \& Barroso, J. (2015). La triangulación de datos como estrategia en investigación educativa. Revista de Medios y Educación(47), 73-88. doi:http://dx.doi.org/10.12795/pixelbit.2015.i47.05

Anastas, P., \& Warner, J. (1998). Green Chemistry Theory and Practice. Nueva York: Oxford University Press.

Anastas, P., Kirchhoff, M., \& Williamson, T. (2001). Catalysis as a foundational pillar of green chemistry. Appl. Catal, 3-13.

Beneitone, P., González, J., \& Wagenaar, R. (Edits.). (2014). Proyecto Tuning América Latina. Meta-perfiles y perfiles. Una nueva aproximación para las titulaciones en América Latina. Bilbao: Universidad de Deusto.

Cann, M. C. (2009). Greening the Chemistry Lecture Curriculum: Now is the Time to Infuse Existing Maistream. En M. C. Cann, Green Chemistry Education. Textbooks with Green Chemistry (págs. 93-100). México.

Cely, V. H., \& Ramírez, M. B. (10 de Noviembre de 2017). Aprendizaje basado en proyectos ecoeficientes y emprendimientos químicos. Congreso Internacional de Investigación, Academia Journals.Com, Online Vol. 9-No. 6. 2017, ISSN 1946-5351. Celaya. Obtenido de Academia Journals.Com.

Cely-Niño, V. H. (2017). Medición de la productividad en procesos industriales que integren cadena de frío, basada en evaluaciones de exergoeconomía y ecoeficiencia. Tesis Doctoral en Ingeniería. Universidad Nacional de Colombia. Bogotá D.C.

Chevallard, Y. (1997). La transposición didáctica: del saber sabio al saber enseñado. Buenos Aires: Aique. 
Colciencias. (2018). Libro verde 2030. Política Nacional de Ciencia e Innovación para el Desarrollo Sostenible.Bogotá D.C: Panamericana.

Creswell, J. (2009). Research Design. Qualitative, Quantitative, and Mixed Methods Approaches. Thousand Oaks (USA): Sage publications Inc, 3nd. ed.

Gómez-Mendoza, M. Á. (2005). La transposición didáctica: historia de un concepto. Revista Latinoamericana de Estudios Educativos, 1(1), 83-115.

González, W. (2018). Aproximación al aprendizaje desarrollador en la educación superior. Educação Santa María, 43(1), 11-26. doi:http://dx.doi.org/10.5902/1984644429309

Hjerensen, D. L., Boese, J. M., \& Schutt, D. L. (2000). Green Chemistry and Education. Journal of Chemistry Education, 77(12), 15-43.

Lederman, N. (2018). La siempre cambiante contextualización de la naturaleza de la ciencia: documentos recientes sobre la reforma de la educación científica en los Estados Unidos y su impacto en el logro de la alfabetización científica. Enseñanza de las Ciencias-Historia y Epistemología, 5(22), 5-22.

Lima, S. (2005). La mediación pedagógica con uso de las tecnologías de la información y las comunicaciones (TIC). La Habana: IPLAC Educación Cubana.

Nueno, P. (2009). Emprendiendo hacia el 2020: Una renovada perspectiva global del arte de crear empresas y sus artistas. Deusto.

Obaya, A., \& Vargas, M. (2013). La Enseñanza Experimental de la Química desde una Perspectiva Interdisciplinaria y Ecológica. En U. U. de, Química Verde Experimental (págs. 27-34). México: Unam.

OECD. Organization for Economic Co-operation and Develop. (2018). Understanding innovative pedagogies: key themes to analyse new approaches to teaching and learning. Work Paper 172. New York: OECD.

Ramírez, M. B. (2018). Encadenamiento mediado por aprendizaje basado en proyectos ecoeficientes. Revista de Investigaciones en Educación $\sum O \Phi I A-S o p h i a$ Educación, 14(2), 60-72.

Ramírez, M. B., \& Cely, V. H. (2017). Formación por competencias del químico colombiano: Encadenamiento a partir del Aprendizaje Basado en Proyectos Ecoeficientes. XXXVIII Congreso Asociación Mexicana de Investigación y Docencia de la Ingeniería Química (págs. 836-838). Ixtapa-Zihuatanejo: AMIDIQ. Obtenido de https://www.dropbox.com/s/7u53cpb23792v44/MEMORIAS\%202017\%20\%28ISBN-978607-95593-5-9\%29.pdf?dl=0

Ramírez, R. (2005). Aproximación al concepto de transposición didáctica. Revista Folios(21), 33-45. doi:https://doi.org/10.17227/01234870.21folios33.45

Segarra, M., Grangel, R., Belmonte, Ó., \& Aguado, S. (2017). ¿Cómo potenciar el emprendimiento y la innovación mediante el desarrollo de proyectos de base tecnológica en el contexto docente universitario? Centro de Estudios Financieros CEF, 11-33.

Soussan, G. (2003). Enseñar las ciencias experimentales: Didáctica y Formación. Santiago de Chile: UnescoEdiciones Antros Ltda.

Tashakkori, A., \& Teddlie, C. (2003). Handbook of mixed methods in social and behavioral research. London: Sage. London: Sage.

Tiberghien, A. (2008). Argumentation in science education: perspectives from classroom-based research. En S. y.-A. Erduran, Foreword. Argumentation in science education: An overview.(págs. ix-vx). Dordrecht, the Netherlands: Springer.

Varela, R. (2010). Creatividad e innovación para el desarrollo del espíritu empresarial. 32, 33-40. 